\title{
Using a simulation game to make learning about angles meaningful. an exploratory study in primary school
}

\author{
Angela Piu ${ }^{1}$, Cesare Fregola2,** and Anna Santoro ${ }^{3, *}$ \\ ${ }^{1}$ Social and Human Sciences Department, University of Valle D'Aosta, Aosta, Italy \\ 2,* University of L'Aquila, L'Aquila, Italy \\ $3,{ }^{*}$ Ministry of Education, Italy \\ For correspondence: a.piu@univda.it
}

\begin{abstract}
As indicated in numerous research studies, schoolchildren encounter many difficulties and obstacles in learning the multifaceted concept of the angle. In order to explore the possibility of enhancing schoolchildren's understanding of such a concept, the authors present a study that aims at investigating some structural characteristics of Angolandia, a simulation game for learning about angles specifically designed according to theoretical-methodological choices for which references were initially drawn from the literature on simulation games and teaching mathematics. This research, involving 31 children from two, 4th-year primary school classes who played Angolandia, reports the observations made as the game was played, an analysis of a written interview on the angle with children before and after the educational activity and an interview with their teachers. These initial data are discussed and considered as the point of departure for systematic experimentation and future developments.
\end{abstract}

Keywords: learning angles, primary school, simulation games, teaching geometry, research.

\section{Introduction}

To understand the angle "object" (Duval R., 2006) students are forced to deal with some difficulties, many or which are related to epistemological obstacles (Brousseau G., 1983) that depend on facts intrinsic to mathematics. The difficulties that arise during the learning process can be traced to a person's semiotic representations of the angle object that are affected by conceptual characteristics permeated by possible "traps" set by mechanisms of perception and by the consequent modalities of representation present in the mind.

In fact, students often have difficulties in understanding that the size of an angle does not vary with the length of the sides and in conceiving of the angle as an open figure. Instead, they tend to consider the angle to be part of the plane limited by two rays and by the arc of circumference relative to the two rays. They often identify it with a specific point or with the "area close" to the vertex, or they talk about a space enclosed between two segments that meet. (Mitchelmore M. \& White P., 2000).

To make the situation even more complex is the presence of various definitions of the angle object, each of which focus on emerging and specific characterisations (D'Amore B., Marazzani I., 2008). In light of such complexity and difficulty, focusing attention on choices, contexts and modalities for using signs that represent the angle can improve student understanding in the attempt to overcome unavoidable "misconceptions" (epistemological obstacles) and to prevent the avoidable ones (educational obstacles, that originate in the teacher's educational and methodological choices), hence giving preference to a gradual means of understanding the concept of angle. 
The authors present an exploratory study that aims at investigating some structural characteristics of a simulation game to introduce the concept of angle and specifically designed according to theoretical-methodological choices for which references were initially drawn from the literature on simulation games and teaching mathematics. The simulation game creates the conditions so that children, acting together, can make decisions to resolve problematic situations using structured materials in compliance with the rules and objectives of the game, and use symbolic representations to communicate, sharing both the methods and the codes to be used.

The authors propose some representations of the angle (Henderson D.W. \& Taimina D., 2005), referred to static and dynamic ones to be introduced into primary school, such as: rotation angle (turning), sector angle (the quantity shared by the set of all superimposed angular sectors), and a pair of half lines that extend from a common point (openness or inclination). They are fully aware, however, that the term "angle" must be considered in relation to the various definitions that have been proposed over time.

The exploratory study is part of a research project in which simulation games for learning geometry are intentionally created, designed, built and tested, and that have been demonstrating their effectiveness. The theoretical and epistemological framework of mathematical learning from which the research has been developed highlights the dialectic nature of the operational dimension and structural processes of the understanding of mathematical concepts (Sfard, 1991) and the role played by everyday language, put in relation to achievable levels of abstraction in the process of construction of mathematical language.

\section{Angolandia}

Angolandia, a simulation game to introduce angles, has been designed for students of the 3rd or 4th year of primary school and sets the following specific learning objectives: to identify the elements and the characteristics of the concept of angle; to recognise and compare angles; to define the angle and its characteristics with one's own words; and to invent and use an economic "code" shared by a group of children to communicate the discoveries relative to the characteristics of the angle made by playing the game.

The game takes place in a village of pinwheels inhabited by dames, knights and elves who like pinwheels because they are so colourful. For the village festival, the dames and the knights decide to decorate the castle with pinwheels of different colours and shapes and ask the great elf to make special pinwheels and to place them in the garden, in the ballroom and in the hall of knights.

So, the great elf begins to make fabulous pinwheels, which he built with a "special tool", and to place them in the castle. Unfortunately, he can't finish the job because an evil wizard made him fall into a deep sleep, from which he could wake up only if someone could finish his work as he had planned...but: "It is impossible!" exclaimed the wizard. Luckily, the great elf left clues in some letters. Playing the part of elves, friends of the great elf, the children, by observing the pinwheels already placed, respectively, in the ballroom and the hall of knights in the castle and in the garden, and by studying the clues in some letters left by the great elf, are asked to discover how he was placing the pinwheels (the garden with pinwheels formed by curved lines, the ballroom with pinwheels formed by congruent angles, the hall of knights with pinwheels formed with different angles), to insert those that they find into the ground and/or to make others and, finally, to write short phrases that represent the groups of pinwheels on the gate of the garden and on the doors of the ballroom and the hall of knights in the castle. 
The children try to solve the problem by comparing each "opening" of each pinwheel by superimposing the "measurement tool" (two "pieces of wood" joined at the end that can remain fixed or turn) that the children find in the great elf's letters.

At this point the elves are invited to discuss how they compared the different pinwheels, and how they made their choices and decided what to write on the doors of the ballroom and the hall of knights and on the gate of the garden, summarising what was done on a sheet of paper that will then be communicated to the wizard, in order to convince him to allow the festivities to continue. In other words, during the game the children are stimulated to invent one or more symbols, i.e., to choose the code, and to remember the similarities that repeat when comparing the pinwheels and their angles. While carrying out these activities, the children decide together how to explain things to the wizard.

The procedural architecture of the simulation games consists of four fundamental phases: - Opening, the initial phase functions to create relational conditions and an appropriate climate for the development of the simulation game; - Briefing, the preparatory phase of the simulation game, during which the objectives of the simulation game are made explicit and instructions for its implementation are provided; - Implementation, the phase of the simulation game itself, during which each participant is placed in a position to act, to experience and to share it, to problematize the reality and to make decisions, to discover the concepts; - Debriefing, the final discussion that allows participants to discuss and share the experience, to systematise the concepts learned and share the geometric language specifically employed.

\section{The game educational material}

The game educational material consists of pinwheels that are flat figures of curved lines without representations of angles (Fig. 1) as well as figures that contain representations of angles, thus making it possible to bring out the elements for which the angles can be distinguished from the comparison with the "non angles".

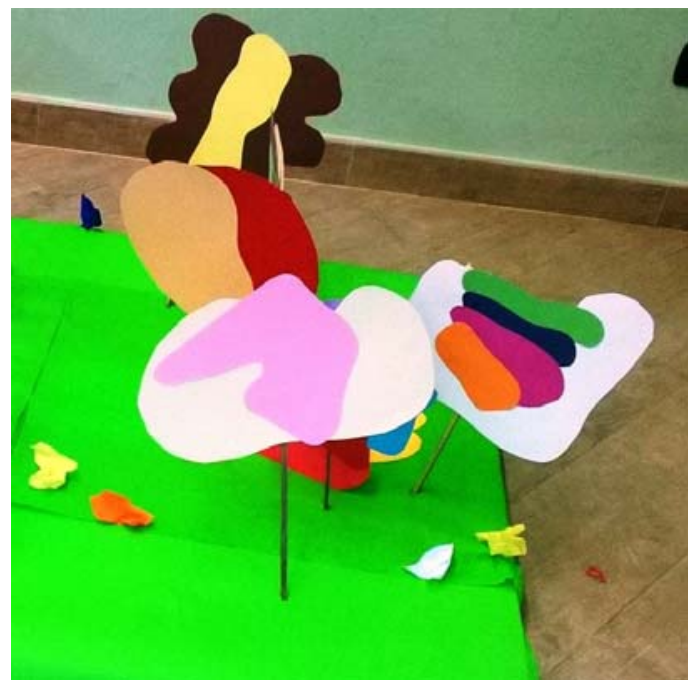

Figure 1. The pinwheels in the garden

The pinwheels with angles consist of consecutive angles that all have a common vertex to cover the size of a turn. The angles have sides with an equal and/or different length (the length of the sides can be different also in the same pinwheel). The pinwheels are made with different coloured fabric or cardboard and are made so that the form of the external part of the pinwheel is irregular or frayed. 
As a result, there are four types of pinwheels with angles: pinwheels consisting of congruent angles (Fig. 2) and pinwheels consisting of different angles (Fig. 3).

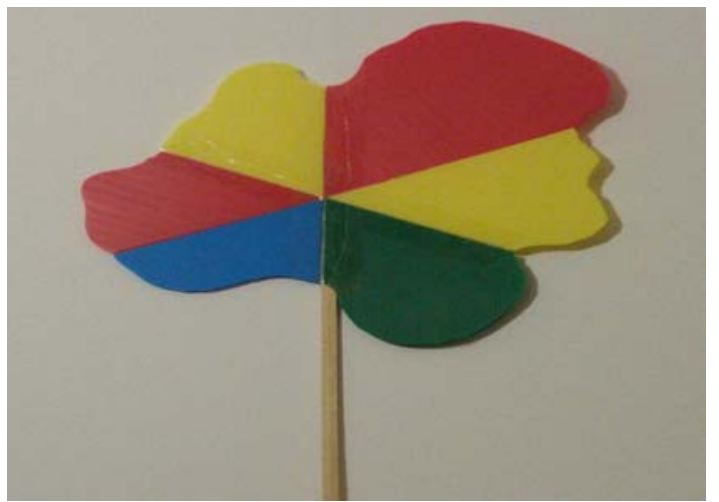

Figure 2. Pinwheels consisting of congruent angles

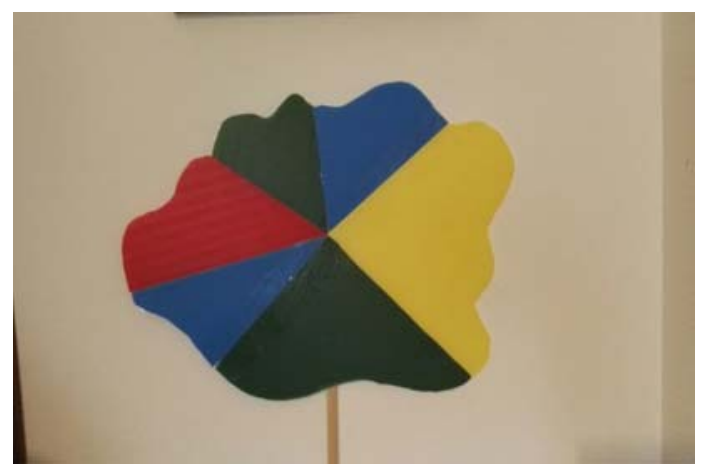

Figure 3. Pinwheels consisting of different angles

For each of the two types of pinwheels similar pinwheels are made and thus with congruent angles. The pinwheels made in this way and the criteria with which the angles are represented can be used to focus attention on the elements of the angle (vertex, sides and size). As a result, the fact that the size of the angle does not vary with a variation in the length of the sides emerges in the angle comparison activities. The immediate perception, by its very nature, is not supported by the comparison and measurement activities. In particular, when comparing pinwheels with congruent angles, consisting of different figures/parts of each pinwheel and of similar pinwheels, it can be noted that, from a perceptive viewpoint, they seem to have a different size even though they are the same size and, thus, are congruent angles.

During the game, along with these static representations of an angle, dynamic representations are also proposed for the pinwheel construction activities so that visual and physical support for the static representation can be integrated with the opportunity of identifying the zero angle or turn and visualising the angles in different positions for the dynamic representation. (Smith C.P. et al., 2014)

Angles are compared using a specially made and unconventional "measurement tool" (Fig. 4) consisting of two pieces of wood joined together in a way that they can remain fixed in a position or rotated. This is used to identify any tricks imposed by perception during the activities to compare congruent angles. The "measurement tool" can be used for the pinwheel construction activities. 


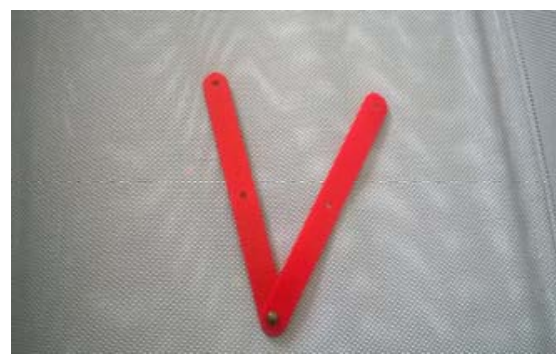

Figure 4. The "measurement tool"

\section{Theoretical framework and methodological choices}

Angolandia complies with the "grammar" of simulation games, i.e., it is structured around a model of reality in which the children act, playing specific roles, making the decisions necessary to solve the intrinsic problematic situations and using specially structured material (Ellington H., 2000; Piu A. \& Fregola C., 2014). Angolandia has peculiar features that derive not only from the theoretical references and from the methodological choices relative to designing simulation games (Piu A. et al., 2015), but also from the choices inherent to the definitions and representation of the angle, based on which the game's educational material was developed. Such choices are taken from recent acquisitions of research in the educational field.

In other words, the model of realty around which Angolandia is designed presents, at a perceptive level, different situations that can be used to represent the concept of angle (Fregola C., 1991; Dienes Z.P., 1971) but without being burdened by non-essential details or by redundant and unnecessary information that could be a source of extraneous cognitive load (Sweller J., 2003) and possibly overload the student and interfere with his/her learning.

Such a model outlines a problematic situation that focuses on the learning task inherent to obtaining the defined learning objectives for Angolandia, and on the mathematizable aspects of angles, thanks to the pinwheels, some of which are made with curved lines and others using angles with an equal and/or different size. To solve the problematic situation, children are asked to do things with their body, hands and gestures using structured material that has been carefully designed and made. These actions induce children to compare figures and to identify the similarities that emerge from such a comparison and that are useful for constructing a representation of the concept.

Along with the actions, the participants are asked to make decisions regarding the means of communication and the codes to be used to come up with a convincing and persuasive explanation for the wizard regarding the solution to the problematic situation, i.e. relative to the comparison of angles and the identification of "non angles" and of equal and different angles forming the pinwheels. In this way Angolandia creates the conditions so that children, acting together, can develop a gradual process of understanding angles, observing and comparing angles, using an unconventional "measurement tool", and making angles in terms of rotation with the same tool, discussing and making comparisons under the guidance of the teacher.

Hence, the children have the opportunity to coordinate the verbal (oral and written) register, as well as the iconic and non-iconic (dynamic) one, the underlying condition for a real differentiation between the concept of angle and its representation and a perquisite to facilitate understanding (Duval R., 2006). So that it can be defined in term of relationship between the development of understanding and the structure of connections between ideas and mathematical procedures in the way in which knowledge is defined and organised (Hiebert and Carpenter, 1992). 
The concrete model proposed and the gradual process create the conditions, therefore, to provide children with an intuitive support that leads them to a significant symbolic representation of mathematical concepts, allowing them to perceive the importance of representation for both understanding and for communicating (Vergnaud, 1994).

In other words, the attention focused on the cognitive and social dimension of learning puts each child in the condition of building new relationships and links starting from the conceptual schemes that they possess, guiding them to integrate old and new schemes and to organise knowledge into compact and structured networks (Anderson, 1983 referenced in Pellerey, 1999) within the context of a shared experience in which "discourses" are developed to re-elaborate such an experience (Pontecorvo, 1983), through a process of distinction-cancellation of important and less important cognitive elements involving the concept being structured.

\section{The transcoding pattern}

The discussion and comparison between children and with the teacher stimulate and orient awareness regarding the use of language, and make it possible for each child to initiate the process of constructing the representations of the angle and using, in making comparisons with others, a semiotic register that starts from the one known and perhaps based on everyday language, in tune with his/her way of representing reality, his/her capabilities and his/her current abstraction skills. In this manner the different levels of meanings that teacher and children understand or attribute starting from one's repertoire of knowledge and experience is the base of a process of developing an intermediate code that can be defined as transcoding, that maintains the object of the communication, the structure, the concept or the rule and hence can constitute a basis for the progressive construction of the mathematical language.

The transcoding pattern is similar to the substantial conception of mathematics, which is considered a language, and understood mainly in terms of its semantic aspect with interest that resides in the meaning underlying the symbols and that considers the formal conception of mathematics an objective of its teaching-learning process.

Within the context of the teaching/learning relationship, the various levels of meanings that teacher and children understand or attribute, starting from one's repertoire of knowledge and experience and during exchanges and interactions, make it possible to construct intermediate and shared codes and to establish the conditions so that the children can: - feel a sense of adequacy in accessing the code that is used and constructed; - perceive the relationship between the words that are used and the understood meanings; - acquire mastery of using a code that is increasingly more formal and always closer and always more similar to the mathematical code.

The condition to be satisfied is to maintain substantial precision during the process of transcoding and constructing a mathematical language, conserving the object of the communication, its structure, the concept that one wants to discover or formalise, and the rule that is applied.

\section{The exploratory study}

The objective of the exploratory study is to analyse whether the characteristics of the structure of a simulation game to introduce the concept of angle and the representations of an angle proposed with the specially constructed educational materials can facilitate understanding of the concept of angle.

The research involved 31 children of two, 4 th year classes of the primary school in the comprehensive institute "Mont Emilius 3" in Charvensod (AO - Italy), and their teacher, who created the scenario of Angolandia and the pinwheels, and who shared the game playing activities with the researchers. 
Angolandia was led by the researcher who proposed the game to three groups of students of the aforementioned classes in October 2015, at separate times in the presence of teachers who acted as observers-participants and as co-leaders during the game. On a rotational basis, the students divided into groups of 10 , with a single group consisting of 11 children, played the game, while the other classmates of a class were involved in other activities outside the classroom and with other teachers.

Before and after the educational experience, children were interviewed and were asked the following question: "Pretend you have to explain to a classmate of another class the meaning of "angle". Write down what you would say". After the experience carried out with the children the teachers and the researcher, on the basis of the observations of the game process and of the analyses of the answers provided by the children, discussed and analysed the experience and the recorded results.

\section{Observation of the game process}

From observations of the three groups of children it emerged that the students who reached the objectives of the game, placing the pinwheels in the respective environments, manipulated the materials and used the measurement tool with precision. All the children fully and actively participated, without interruptions.

Observations revealed that the number of dialogues, the interactions and the actions using the tool to compare angles and to build them, involved each child.

For the three groups, the average time for presenting the scenario and explaining the problematic situation was 10 minutes, while the duration of the simulation game, excluding the debriefing part, and considering the average time used by the three groups, was calculated to be around 60 minutes. Instead, the debriefing lasted 15 minutes.

For each stage of the game, the systematic observations made generated the following information: In the initial stage, the children of all three groups verbalised the difference between the pinwheels located in the garden and those in the two rooms of the castle, i.e., between the pinwheels consisting of curved lines and those consisting of representations of angles. The different groups formulated, instead, a series of hypotheses about the similarities between the pinwheels consisting of angles placed in each environment (respectively, pinwheels with equal angles and pinwheels with different angles) that were not noted in the material already positioned in the halls of the castle. Their attention, in fact, focused on the difference in colour, on the different overall dimensions of the pinwheels and on the difference in the shapes of each pinwheel.

The leader, after giving the children time to discuss the aforementioned hypotheses, helped them to find the letter containing the clues with which the great elf had decided to insert the pinwheels in the various environments, and the "measurement tool" that he used. After finding and reading the letter, the children began to use the tool to compare the parts of the pinwheels and identified and verbalised the similarities present in the pinwheels inserted in the ballroom (pinwheels with equal angles) and in the knights' hall (pinwheels with different angles), while they did not find similarities between the groups of similar pinwheels (Fig. 5). The discovery of similarities confirmed after checking using the "measurement tool" allowed the children to review some of the hypotheses that were previously formulated and influenced by an examination based exclusively on perception.

During such an activity, each group brought up references to subjects that can be traced to the children's experiences and that were solicited by the structure of the game: in one group an analogy emerged with the optical illusions, understood as illusions that play tricks on the human visual apparatus, making the group incorrectly perceive something that in realty is something else; in another group a reference to similar figures emerged, understood not only according to a mathematical meaning, but in daily use, such as figures that have some features that are the same and 
others not. The reference in this case was not to the similar pinwheels in Angolandia, but instead to the pinwheels with equal angles, in which similar "aspects" were noted (the congruence between angles) as well as different ones (the shape of the figures and the colours); the third group, instead, focused its discussion on the variations in the opening of the parts of the pinwheels that start from the centre, showing how the closer the sides get to each other the more they form "pointed figures" and vice versa.

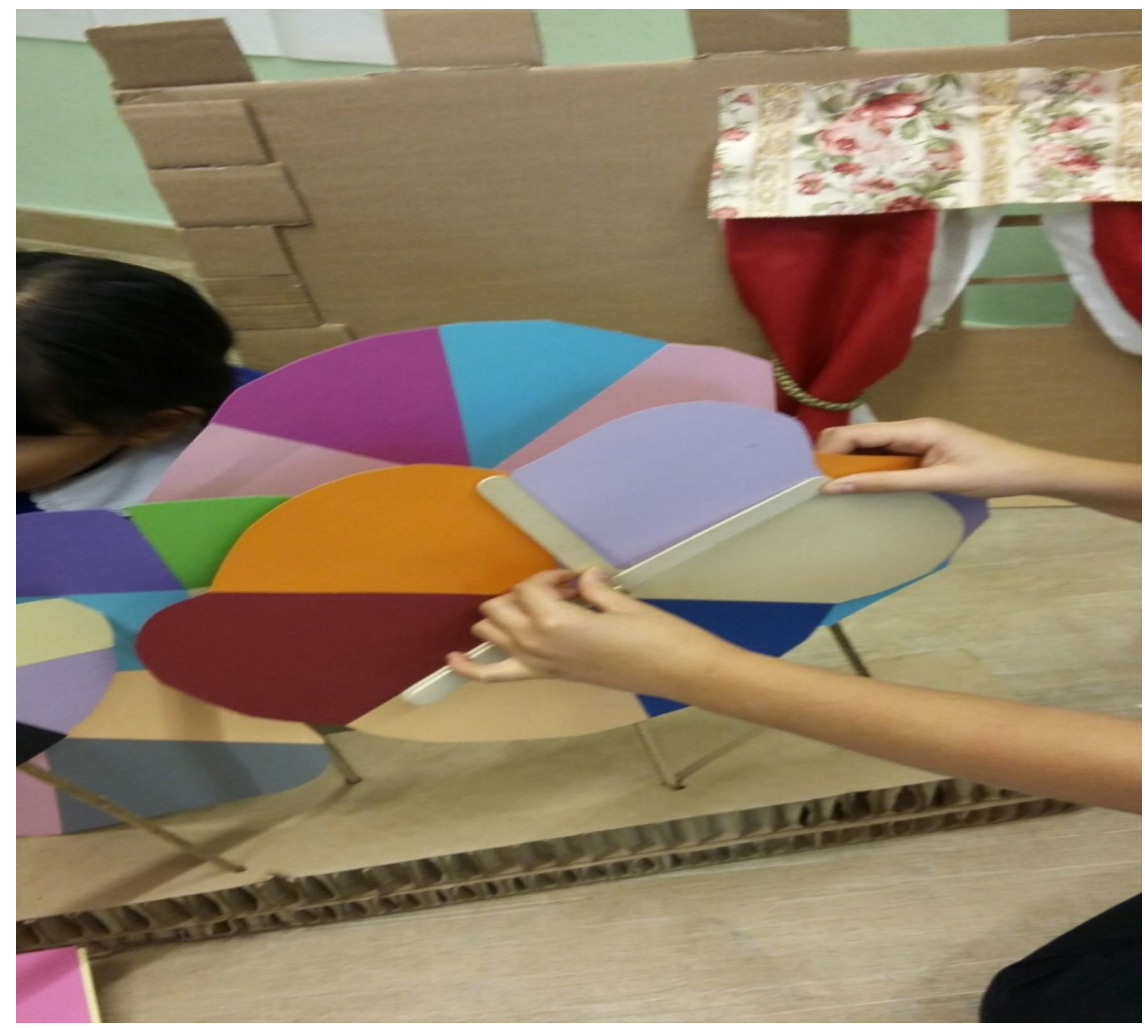

Figure 5. The children use the tool.

Following this discovery, the children began to scatter the pinwheels on the ground, respectively, in each environment. After completing this activity they began to build, at their discretion using cardboard, different pinwheels to put in the respective environments using rotation the "measurement tool". Some of them also made pinwheels without angles, drawing them with a pencil on the cardboard. After this activity they decided together what to write in the phrases that they made and positioned.

At the end of the game, the children participated in the final discussion led by the researcher. Together, they referred to the experience during the game, with particular reference to the similarities found in and between the pinwheels and referring to the themes of discussions that emerged. On the basis of such references, the group organised together the characteristics of the angle that arose while playing the game and shared the code and the meanings that emerged.

\section{Analysis of children's phrases about the angle}

Two types of analyses were carried out relative to the interview with children. One involves the answers provided by children to identify the types of responses that describe different ways of representing the concept of angle after the educational activity. In other words, the meanings attributed by the children to the mathematical concepts with natural language and the meanings expressed by the children that can be inferred, considered in relation to the representation of the concept. The second analysis refers to the comparison between the answers provided prior to the 
educational activity and those provided by each child after playing Angolandia, to identify how the representation of the concept being developed evolves.

With reference to the first analysis and to the characteristics of the concept of angle, the categories of analyses that emerged from the variety of definitions provided by the children, after the educational activity, and identified by the two researchers, with a more than $80 \%$ agreement out of the total of answers examined, are the following:

Vertex. Definitions by children that refer only to the vertex. Examples of phrases in this category are: "The angle is a point"; "An angle is a vertex".

- $\quad$ Sides and vertex. Definitions by children that indicate that the angle consists of two lines that meet around a point. Examples of phrases belonging to this category are: "An angle is a meeting point between two lines and the point of the meeting is called vertex"; "The angle is represented by a vertex and two half lines".

- $\quad$ Sides, vertex and opening. Definitions by children indicating that the angle consists of two lines that meet in a point, making some attempt to express an angular relationship between the lines (using words such as opening, turn, space, area, gap, distance, size and measurement). Examples of phrases belonging to this category are: "The angle is a corner. The opening is called a half line, and the starting point the vertex"; "The angle is all the space that there is for example between one stick and another".

Two subcategories were identified within this category: one that contains definitions for which the discussion focuses on the formation of two portions of a plane/space and the other containing definitions that bring out the concept of unlimitedness. Examples of answers belonging respectively to the following subcategories are:

- for the first one: "The angle is a meeting place between two lines that meet forming two sides that divide the spaces"; "An angle is the space found between two lines. The one outside the two lines is another larger angle"; the student clarifies this definition by showing a drawing that represents the one being described.

- For the second subcategory, instead, examples are: "The angle is a space that begins from the vertex"; "I would say that you don't have to see if one angle is larger or smaller. It's enough to see if there is an opening".

- Vague responses. Named or that were vague to the point of being incomprehensible.

With respect to the second analysis, considering that not all children gave a definition of angle in the written interview prior to the activity, some categories were identified by comparing the definitions that each child gave before and after playing Angolandia. With an agreement of more than $80 \%$ of the total of the answers examined among the two researchers, those categories are:

- Unvaried definitions, i.e., for which no significant differences emerge in relation to the representation of angle. Examples are: from "An angle is a corner" to "An angle is a vertex"; from "An angle are two lines that meet and stop" to "The angle means two lines that meet".

- Definitions that indicate an integration/restructuring of the representation of the concept of angle with respect to the previous definition. These include the following four subcategories:

- $\quad$ From segments/sides - to vertex and sides: Examples are: from "An angle is the sides of a house" to "An angle has sides and vertices";

- $\quad$ From corner - to opening: from "The angle is a pointed corner" to "I would say that you don't have to see if one angle is larger or smaller. It's enough to see if there is an opening".

From intersection - to opening: from "An angle is like the place in a table where two lines meet" to "The angle is a meeting place between two lines that meet forming two sides that divide the spaces"; from "The angle is the pointiest part of the table. Or a broken line." to "The angle is the part in the middle of two traced lines".

From closed space/limitedness - to open space, unlimitedness. Examples are: from "The angle are two lines that form a half a square" to "The angle is a space that begins from the vertex". 


\section{Interview with teachers}

The interview with teachers held after the activity with the children focused on what the children had learned and on the differences with respect to previous activities carried out by the teachers to introduce the concept of angle. Compared to other types of activities, such as lessons and activities using the fan, change of directions with toy cars, use of paper, clock and an IWB, the teachers stated that the activity was "economical", meaning that it can be used to understand the representation of the concept of angle without having to carry out different activities. For them there are significant and varied activities that can be used to complete the introduction to the concept of angle. From an informal evaluation carried out in their classes after the educational activity and before continuing with other planned activities about the angle, the teachers received confirmation that all children had understood something about the angle. Indeed, the children told their teachers that they particularly appreciated the angle construction activities during the game. Along with the economic factor, the teachers noted the greater importance of the perceptive and dynamic aspect that emerged from the proposed didactic material compared to other educational activities.

Based on an analysis of the written interviews of the children about the meaning of angle discussed with the researcher, the teachers were positively impressed by some of the children's answers, in particular those that identified the two portions of a plane and those from which the meaning of unlimitedness of an angle could be inferred.

With regard to the game process, the researcher, along with the teachers, retracing the process involved in playing Angolandia, noted the importance of the "measurement tool", as emerged from observations of the children belonging to the three groups, as an effective support for identifying the criteria with which the great elf placed the pinwheels in the halls and for making comparisons useful for determining the similarities that could be used to initiate the process of constructing the concept and of supporting the discussion among the children.

\section{Conclusions and future developments}

In this article the authors presented an exploratory study designed to investigate if the characteristics of the structure of a simulation game to introduce the concept of angles and the representations of an angle can facilitate the processes for understanding the concept of angle.

The game called "Angolandia", which involved the participation of 31 children in the 4th year of the primary school in the comprehensive institute "Mont Emilius 3" in Charvensod (AO - Italy) along with their teachers, was proposed within the context of a research project about the characteristics and the structure of simulation games. During the study, the underlying process of the simulation game, for which three separate groups of children participated, was observed, the meanings about the angle expressed by the children in a written form before and after the educational experience were analysed, and an interview was held between the researcher and the teachers to discuss the game process and the phrases written by the children. The aforementioned analyses made it possible to identify some significant aspects, including:

- the richness and variety of definitions of angle provided by the children at the end of the game, for which, among others, the most important were the identification of two portions of a plane and the concepts of unlimited plane;

- the measurement tool used in the Angolandia simulation game, that supported the children's actions, within the transcoding pattern, understood as a mediator between the children's discussions with each other, the angle "object" and the geometric language that expresses it. 
Hence, thanks to the simulation game, the children were able to explore, invent and provide some representations of an angle, associating language, its meanings and the definitions of a complex concept with multiple levels of analysis, applications and "challenges" for learning geometry.

It is based on these aspects, considered positive indicators of game functionalities and of the effectiveness of the specially designed materials that the children were able to use, that future research proposals have already been established. Bearing these proposals in mind, the discussions between children guided by the teacher during the game will be analysed and the understanding and the definitions of the angle "object" will be investigated in greater depth so as to obtain additional study elements inherent to the semiotic choices implemented in designing the educational process.

\section{Acknowledgements}

Special thanks to the pupils and their teachers who partecipated so enthusiastically in this research.

\section{References}

Anderson, J. R., (1983). The architecture of cognition. Cambridge, MA: Harvard University Press.

Brousseau, G., (1983). Les obstacles épistémologiques et les problèmes en mathematiques. Recherches en didactique des mathématiques. 4(3), 165-198.

D'Amore, B., Marazzani, I., (2008). L'angolo, oggetto matematico e modello spontaneo. La matematica e la sua didattica. 22(3), 285-329.

Dienes, Z. P., (1971), Le sei tappe del processo d'apprendimento in matematica. Firenze, Italy: OS.

Duval, R., (2006). A cognitive analysis of problems of comprehension in a learning of mathematics. Educational Studies in Mathematics, 61(1), 103-131.

Ellington, H., (2000). Games and simulations - media for the new millennium. In Saunders D., Smalley, N. (Eds.), The International simulation and gaming research yearbook. Vol. 8, pp. 13-32. London: Kogan Page.

Fregola, C., (1991). Geometria, Logica, Probabilità e Informatica. In Laeng, M., (Ed.), Percorsi Didattici. Teramo, Italy: Lisciani Editore.

Henderson, D.W. and Taimina, D., (2005). Experiencing geometry. Euclidean and non-Euclidean with history. New York: Cornell University.

Hiebert, J. and Carpenter, T. P., (1992). Learning and Teaching with Understanding. In Grouws, D. A. (Ed.). Handbook of Research on Mathematics Teaching and Learning. New York: Macmillan, pp. 65-97.

Mitchelmore, M. and White, P., (2000). Development of angle concepts by progressive abstraction and generalisation. Educational Studies in Mathematics, 41(3), 209-238.

Pellerey, M. (1999). Le conoscenze matematiche. In Pontecorvo, C., (Ed.), Manuale di psicologia dell'educazione. Bologna, Italy: Il Mulino, p. 221-241.

Piu A., Fregola C., (2014). Transcoding Pattern and Simulation Games in Learning Geometry. A Research in Primary School. In Meijer S.A., Smed R., (Eds). Frontiers in Gaming Simulation (p. 21-28). Switzerland: Springer International Publishing.

Piu, A., Fregola, C., Santoro, A., (2015). Development and Analysis of a Design Model for Geometry-Based Simulation Games, Procedia - Social and Behavioral Sciences, 186, 293-304.

Pontecorvo, C., (Ed.). (1993). La condivisione della conoscenza. Firenze, Italia: La Nuova Italia.

Sfard, A. (1991). On the dual nature of mathematical conceptions: reflections on processes and objects as different sides of the same coin. Educational Studies in Mathematics, 22, 1-36.

Smith, C.P., King, B., Hoyte, J. (2014). Learning angles through movement: Critical actions for developing understanding in an embodied activity. The journal of mathematical behaviour, 36, 95-108

Sweller, J., (2003). Evolution of human cognitive architecture. In Ross, B. (Ed.). The Psychology of Learning and Motivation. 43, (pp. 215-266). San Diego: Academic Press.

Vergnaud, G., (1994). Le rôle de l'enseignement à la lumière des concepts de schème et de champ conceptuel. In Artigue M., Grass R., Laborde C. et Tavignot P., Vingt ans de didactique des mathématiques en France (p. 177-191). Grenoble, France: La Pensée Sauvage. 